\title{
A importância da liga acadêmica na promoção do envelhecimento saudável
}

\section{The importance of the academic league in promoting healthy aging}

\section{La importancia de la liga académica para la promoción del envejecimiento saludable}

\author{
Shaumin Vasconcelos Wu iD \\ Universidade do Estado do Pará - Belém (PA) - Brasil
}

Camila Alcântara Fernandes (D)

Universidade do Estado do Pará - Belém (PA) - Brasil

Dayane Cereja Ferreira da Silva (iD

Universidade do Estado do Pará - Belém (PA) - Brasil

Elissa Raissa Siqueira do Nascimento (iD

Universidade do Estado do Pará - Belém (PA) - Brasil

Jamylle Silva Campos (iD

Universidade do Estado do Pará - Belém (PA) - Brasil

João Sérgio de Sousa Oliveira (iD

Universidade do Estado do Pará - Belém (PA) - Brasil

\section{RESUMO}

Objetivo: Relatar a experiência de discentes de uma liga acadêmica de Fisioterapia em gerontologia em uma ação para promoção do envelhecimento saudável. Síntese dos dados: Trata-se da descrição da experiência de discentes de Fisioterapia em uma ação organizada pela Liga Acadêmica de Fisioterapia em Gerontologia (LAFIGE), vinculada à Universidade do Estado do Pará, na IV ação de extensão da liga, intitulada "Promoção do envelhecimento saudável", vivenciada em uma praça pública de Belém, Pará, Brasil. Nessa ação, participaram da organização: 13 membros da LAFIGE, sendo 12 acadêmicos e um orientador, e 50 participantes com idade igual ou superior a 20 anos. Antes da ação, o coordenador repassou as orientações aos discentes acerca de orientações (sob supervisão profissional) de condicionantes do envelhecimento saudável, avaliações físico-funcionais, exercícios físicos e incentivo à alimentação saudável. Tal experiência da ação possibilitou a troca de informações entre a comunidade e a academia, além de favorecer a educação em saúde da população acerca do processo de envelhecimento e da importância de preservar a capacidade funcional. Ademais, incentivou-se a construção de aprendizados científicos e interpessoais de forma bidirecional, entre os acadêmicos e participantes, enfatizando a importância da inserção da fisioterapia em gerontologia na promoção da saúde no envelhecimento. Conclusão: Observou-se a essencialidade da prática de educação em saúde em busca de uma velhice saudável, com a conscientização da população sobre o processo de envelhecimento, além de uma adequada capacitação dos ligantes envolvidos, incentivando ações que visem à promoção da saúde dos idosos.

Descritores: Educação em Saúde; Envelhecimento; Conscientização; Fisioterapia; Geriatria.

\section{ABSTRACT}

Objective: To report the experience of students of a Physiotherapy in Gerontology Academic League in an action taken to promote healthy aging. Data synthesis: This study describes the experience of students in an action organized by the Physiotherapy in Gerontology Academic League (Liga Acadêmica de Fisioterapia em Gerontologia - LAFIGE) of the Pará State University and put into effect in the IV extension act promoted by the league, which was titled "Healthy Aging Promotion" and held in a public square in Belém. Pará, Brazil. Organizers of this action were: 13 LAFIGE members - 12 students and 1 tutor - and 50 participants aged 20 or over. Before the action, the coordinator briefed the students with guidelines (under professional supervision) on healthy aging conditioners, physical and functional assessments, physical exercises and promotion of healthy eating. Such experience with the action enabled the exchange of information between the community and the academia and favored the promotion of health education across the population with regard to the aging process and the importance of preserving functional capacity. Furthermore, the construction of scientific and interpersonal learning in a bidirectional way was encouraged between the students

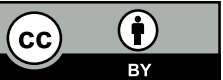


and the participants, with emphasis on the importance of the insertion of the physiotherapy in gerontology in order to promote health during aging. Conclusion: Health education practice is essentially needed for a healthy aging and for raising people's awareness of the aging process. In addition, there is a need for adequate training of the league members involved actions aimed at promoting the health of the older adults should be encouraged.

Descriptors: Health Education; Aging; Awareness; Physical Therapy Specialty; Geriatrics.

The Universal Trial Number (UTN): U1111-1240-0408

\section{RESUMEN}

Objetivo: Relatar la experiencia de estudiantes de una liga académica de Fisioterapia en gerontología en una acción para la promoción del envejecimiento saludable. Síntesis de datos: Se trata de la descripción de experiencia de estudiantes de Fisioterapia en una acción de la Liga Académica de Fisioterapia en Gerontología (LAFIGE) que está conectada con la Universidad del Estado de Pará en la IV acción de la liga intitulada "Promoción del envejecimiento saludable" que ha sido vivenciada en una plaza pública de Belém, Pará, Brasil. Para la acción han participado de la organización 13 miembros de la LAFIGE de los cuales 12 académicos y uno tutor y 50 participantes con la edad igual o mayor de 20 años. Antes de la acción el coordinador ha hablado con los estudiantes sobre las orientaciones (bajo la observación de un profesional) de los condicionantes del envejecimiento saludable, las evaluaciones físico-funcionales, los ejercicios físicos y el incentivo para la alimentación saludable. La experiencia de la acción ha permitido el cambio de informaciones entre la comunidad y los académicos además del beneficio de la educación en salud para la población sobre el proceso de envejecimiento y de la importancia del mantenimiento de la capacidad funcional. Además, la experiencia ha incentivado la construcción de aprendizajes científicos e interpersonales de manera bidireccional entre los académicos y los participantes con énfasis para la inserción de la fisioterapia en la gerontología para la promoción de la salud en el envejecimiento. Conclusión: Se observó la esencialidad de la práctica de educación en salud para la vejez saludable con la concienciación de la población sobre el proceso de envejecimiento además de una capacitación adecuada de las personas involucradas para incentivar acciones para la promoción de la salud de mayores.

Descriptores: Educación en Salud; Envejecimiento; Concienciación; Fisioterapia; Geriatría.

\section{INTRODUÇÃO}

O mundo vem passando por um processo de transição demográfica em uma velocidade surpreendente, emergindo como uma questão política importante ${ }^{(1)}$. Projeções mundiais da Organização Mundial da Saúde (OMS) mostram que a população idosa irá praticamente dobrar, passando de 1 bilhão em 2019 para cerca de 2,1 bilhões em $2050^{(2)}$. O ritmo desse processo não é homogêneo, uma vez que alguns lugares, como o Brasil, terão pouco mais de 20 anos para fazer a mesma adaptação que outros países, que levaram décadas ${ }^{(3)}$.

No ano de 2020, o Brasil apresentou um contingente populacional de pessoas com idade igual ou superior a 60 anos estimado em 30 milhões. Entre os estados da região Norte, destaca-se o Pará, com 793.740 habitantes idosos $^{(4)}$. Na capital, Belém, ocorre a maior concentração, com aproximadamente 257 mil idosos (17,1\% da população), o que a torna uma cidade demograficamente envelhecida ${ }^{(5,6)}$. Esses cenários, em nível global, levaram a OMS a elaborar a Década do Envelhecimento Saudável (2020-2030), oportunizando a reunião de diversos setores para o desenvolvimento de ações rápidas e colaborativas para melhoria da vida dos idosos, familiares e comunidade(2).

Nesse cenário, nota-se que o prolongamento da vida é uma aspiração de qualquer sociedade, porém esse processo de envelhecimento populacional, por vezes, não ocorre com qualidade de vida ${ }^{(7)}$. A concepção de saúde para o idoso está intimamente ligada à independência e autonomia, sendo assim, um idoso com capacidade funcional é aquele que possui a habilidade de gerenciar sua vida, tomar decisões e realizar atividades cotidianas com os próprios meios. No entanto, nessa fase, há alterações morfofisiológicas características, que frequentemente declinam algumas funções, podendo resultar em limitações físicas, funcionais e emocionais ${ }^{(8,9)}$.

Associado a esse contexto, a destinação de atenção à pessoa idosa em nível comunitário impõe aos profissionais e acadêmicos das ciências da saúde conhecimentos norteados por políticas públicas que envolvam The Global Strategy and Action Plan on Ageing and Health 2016-2020, a Política Nacional de Saúde da Pessoa Idosa e a Política Nacional do Idoso. Além dessas, torna-se essencial a contextualização na Política Nacional de Promoção da Saúde, que contempla entre os seus valores e princípios a solidariedade, a felicidade, o respeito à diversidade, a humanização, a inclusão social e a integralidade ${ }^{(10,11)}$.

Atualmente, na atuação do profissional de Fisioterapia, esses conhecimentos são fortalecidos pelo reconhecimento nacional em resolução que reconhece e disciplina a especialidade profissional da Fisioterapia em gerontologia ${ }^{(12)}$. No meio acadêmico, o tripé ensino-pesquisa-extensão é a base da produção e estimulação de conhecimentos sobre 
o processo de envelhecimento, além de subsídios ao planejamento e execução de ações de promoção da saúde e do envelhecimento saudável(10,13-15).

Esse meio acadêmico utiliza, atrelado a esse tripé, as ligas acadêmicas, sob orientação de um professor vinculado a instituição de ensino superior, que buscam preencher lacunas de conhecimento em temáticas específicas ${ }^{(16)}$. Segundo a Sociedade Brasileira de Geriatria e Gerontologia (SBGG), as ligas relacionadas ao envelhecimento devem ter em seus objetivos a mudança da concepção pessimista e de insegurança emocional sobre o processo de envelhecimento, por meio de diversas atividades extracurriculares baseadas no tripé acadêmico, visando não somente à divulgação de informações para a promoção de saúde às pessoas idosas, mas também à integração da comunidade com os acadêmicos ${ }^{(17)}$.

Nesse sentido, a atuação da Fisioterapia em gerontologia, para manutenção e promoção de saúde e qualidade de vida, busca contemplar não somente as múltiplas dimensões do envelhecer, mas também percebe o indivíduo como ser humano autônomo, que possui a necessidade de executar atividades cotidianas e laborais por vezes, bem como participar do contexto que o envolve ${ }^{(18)}$. Considera também o movimento humano, utilizando a prática de exercício físico para proporcionar melhorias nas funções orgânicas, na capacidade funcional e na integração social e cultural ${ }^{(19,20)}$. Além da educação em saúde, é uma ferramenta importante de empoderamento de cuidados necessários à promoção de um envelhecimento saudável, pois é entendida como prática para a transformação dos modos de vida dos indivíduos e da coletividade ${ }^{(9)}$.

Assim sendo, a promoção da saúde mostra-se fundamental por ações que permitam vivências e trocas de experiência entre os atores envolvidos, na busca de uma percepção holística dos pilares que envolvem a gerontologia e o uso da Classificação Internacional de Funcionalidade, Incapacidade e Saúde (CIF) na atuação fisioterapêutica com a comunidade. Nesse contexto, o presente artigo tem como objetivo relatar a experiência de discentes de uma liga acadêmica de Fisioterapia em gerontologia em uma ação para promoção do envelhecimento saudável.

\section{SÍNTESE DOS DADOS}

Trata-se de um relato de experiência a partir das vivências práticas de discentes de Fisioterapia por uma ação organizada pela Liga Acadêmica de Fisioterapia em Gerontologia (LAFIGE), vinculada à Universidade do Estado do Pará como atividade extensionista pela Pró-reitoria de Extensão (PROEX), além de ser reconhecida e cadastrada na Sociedade Brasileira de Geriatria e Gerontologia (SBGG). A ação foi organizada e realizada por uma equipe composta por 13 membros da LAFIGE, sendo 06 diretores da liga, 06 ligantes efetivos da instituição e um professor orientador da liga.

A IV ação de extensão da LAFIGE intitulada Promoção do envelhecimento saudável, foi realizada no dia 4 de junho de 2017, na praça Batista Campos, localizada na Região Metropolitana de Belém, Pará, durando cerca de quatro horas. A amostra foi constituída por 50 participantes, na faixa etária de 20 a 75 anos, selecionados por conveniência, por meio de recrutamento da população que estava presente no local e horário da ação.

Foram incluídos maiores de 18 anos, de ambos os sexos, que teriam disponibilidade de tempo para realização das atividades. Havia adultos, uma vez que a temática do processo de envelhecimento é inerente a todas as pessoas desde o nascimento, sendo imprescindível o conhecimento precoce sobre o seu contexto e cuidados necessários na atenção à saúde, visando ao envelhecimento saudável. Excluíram-se participantes que apresentaram incapacidade funcional para as atividades propostas, estivessem em jejum, com pressão arterial e/ou frequência cardíaca alteradas e/ou com alterações cognitivas pelos padrões do miniexame do estado mental ajustado pela escolaridade. Um ponto que merece ser ressaltado é a não inclusão de jovens, pois, apesar da ação ter sido realizada em praça pública, não houve aviso prévio aos seus frequentadores e o protocolo da ação exigia assentimento de seus pais/responsáveis, por serem menores de idade.

Realizou-se o planejamento da ação em duas etapas: primeiramente, ocorreu a idealização do tema e a revisão da literatura no mês de maio. Posteriormente, realizou-se a capacitação da equipe de trabalho e a socialização de artigos científicos acerca da temática com os participantes da liga. Abordou-se a estrutura e o planejamento da ação em saúde, assim como: a padronização de verificação da pressão arterial sistêmica pela $7^{\text {a }}$ Diretriz Brasileira de Hipertensão Arterial(21); a mensuração da altura e do peso o cálculo do índice de massa corporal (IMC) e as orientações alimentares no contexto dos cuidados básicos em saúde pelos critérios da $\mathrm{OMS}^{(22)}$; a classificação da funcionalidade considerando as premissas da CIF, além dos domínios de mobilidade, cognição humor e comunicação(23); as condutas cinesioterapêuticas, considerando as Recomendações Globais de Atividade Física para Saúde da OMS, além das recomendações para Atividade Física e Saúde Pública para Adultos Idosos do Colégio Americano de Medicina do Esporte e da Associação Americana do Coração(24). 
A segunda etapa desenvolveu-se no dia da ação, sendo dividida em três momentos. No primeiro, as orientações aos participantes sobre os fatores determinantes e condicionantes, pois são fundamentais ao envelhecimento saudável(25). No segundo, houve a aferição de pressão arterial e a mensuração da altura, peso e IMC, momento que receberam uma carteirinha contendo os dados mensurados para controle da sua saúde e seguimento dos cuidados. E, no terceiro, houve a realização de atividades físicas, com uma duração média de 20 minutos, iniciados por 5 minutos de aquecimento, seguidos por alongamentos dos principais grupos musculares de membro superior e inferior e 10 minutos de atividades funcionais (agachamento; avanço; jogar e agarrar bolas pequenas; treino de sentar e levantar na bola suíça; treino de agilidade por deslocamentos laterais e progressivos utilizando cones pequenos) e treino cognitivo utilizando-se um tapete com cores (azul, verde, amarelo e vermelho), no qual os participantes deveriam pisar, de acordo com o comando verbal da cor, com intensidades e repetições adaptadas para cada pessoa de acordo com a percepção do terapeuta e dos participantes ${ }^{(26)}$.

A educação em saúde divide-se em duas formas: a tradicional e a dialógica. A primeira diz respeito ao modelo preventivo, com foco na doença e em uma intervenção curativista; enquanto a segunda classificação tem como princípio a promoção de saúde, caracterizando-se pelo diálogo bidirecional entre os participantes, ou seja, os profissionais e a população, permitindo ganhos para ambas as partes ${ }^{(27)}$.

Como benefício ao público em questão, pode-se citar a relevância na formação dos grupos entre os participantes, o que propiciou a construção de conhecimentos e aprendizados e de novas habilidades, essenciais para o fortalecimento da identidade ${ }^{(28)}$. Somado a isso, entende-se que é inerente à educação em saúde o planejamento e desenvolvimento de estratégias educativas, as quais intervenham no processo saúde-doença da população, permitindo um viver com mais bem-estar ${ }^{(29)}$.

Assim, a LAFIGE promoveu uma ação voltada ao envelhecimento saudável da população em diferentes faixas etárias, haja vista a necessidade de abordar o tema precocemente. Com o auxílio de um folder foi possível aprofundar questões sobre promoção do envelhecimento saudável, sendo explorada a importância do exercício físico, da alimentação adequada, da realização de atividades de lazer, entre outros, o que reforça a importância da educação em saúde na promoção de um olhar de conscientização sobre a própria saúde ${ }^{(29)}$.

Nesse sentido, o contato maior com a população permitiu que o conhecimento fosse bidirecional, entre os acadêmicos e participantes. Esses resultados se mostram coerentes com a literatura, entre eles, um estudo que desenvolveu semanalmente atividade de educação em saúde com um público de sessenta idosos, sendo possível observar que essas ações são fundamentais para fortalecer vínculos, visto que os idosos, durante os grupos, podem partilhar das mesmas experiências e dividir suas angústias ${ }^{(30)}$.

Ademais, nota-se a importância dos profissionais da saúde nesse contexto, tendo em vista que possuem um papel contínuo no aumento do alcance à informação de qualidade. O fisioterapeuta em gerontologia possui a competência de atuar em todos os níveis de atenção à saúde. Sua intervenção na promoção e prevenção traz múltiplos benefícios, pois a ele são peculiares algumas atividades, tais como: avaliações das funções musculoesqueléticas, bem como ergonômicas; realização de prognóstico fisioterapêutico e estabelecimento de orientações a familiares e cuidadores ${ }^{(31)}$. Todavia ressalta-se que a educação em saúde não é uma via de mão única, evidenciando o impacto positivo também para quem propõe essas ações ${ }^{(29)}$.

Pode-se, a partir da ação, proporcionar aos acadêmicos um contato com a comunidade, fortalecendo a extensão universitária. É importante mencionar que ela age como um relevante processo cultural, interdisciplinar e de grande interação dialógica, permitindo uma formação mais humanista e crítica(32).

Dentre os aspectos relevantes observados, destaca-se a percepção da satisfação dos idosos em dividir histórias e dar exemplos, tornando esses momentos válidos por promoverem a troca educativa e permitirem que os acadêmicos exerçam a prática da escuta ${ }^{(33)}$. A comunidade obteve esclarecimentos sobre o presente tema e, por conseguinte, recebeu avaliações antropométricas para incentivar o cuidado com o corpo. Aliado a isso, no momento da avaliação, os participantes eram informados sobre os resultados da pressão arterial, altura, peso e IMC para instigá-los na maior atenção à saúde.

Todavia vale ressaltar que a atividade não se pautou meramente na transmissão de informações. As orientações repassadas levaram em consideração o contexto de cada participante, visto que as características físicas, emocionais e sociais são de suma importância ${ }^{(34)}$. Tal fato é de extrema relevância, uma vez que se encontra atrelado à Política Nacional de Saúde, a qual afirma que é preciso compreender o envelhecimento saudável, termo adotado pela Organização Mundial da Saúde, como uma vivência rica e positiva, a qual segue acompanhada por pilares essenciais de saúde, envolvendo os diversos aspectos do cotidiano do adulto/idoso(35).

Em relação ao terceiro momento da atividade, com a prática do exercício físico pôde-se, de forma dinâmica, chamar atenção para o papel fundamental do condicionamento físico para um envelhecer com saúde, estimulando-os 
a práticas que os tornem mais ativos e que proporcionem benefícios funcionais. A execução de exercícios físicos, além de proporcionar melhora na amplitude de movimento articular, também têm sido recomendada como meio de atenuar os efeitos deletérios do processo de envelhecimento ${ }^{(36)}$. Ao término das atividades eram oferecidas frutas aos participantes, ressaltando outra questão altamente relevante, a alimentação saudável.

Nota-se que a associação de alterações comuns da senescência ao uso de medicamentos aumenta o risco de uma nutrição não balanceada, além do aparecimento de doenças que podem atrapalhar todo o processo de ingestão, digestão e absorção dos nutrientes, o que compromete ainda mais o estado de saúde do idoso ${ }^{(37)}$. Desse modo, é perceptível a importância de se atentar para esse assunto e abordá-lo da melhor forma possível.

A associação da polifarmácia com uma má nutrição, em especial na população idosa, pode corroborar para o desenvolvimento de um fenômeno cada vez mais comum e preocupante, a sarcopenia. Ela é definida como a redução progressiva da massa e força muscular do indivíduo, implicando, consequentemente, em impactos negativos à funcionalidade do idoso ${ }^{(38)}$.

Outro agravante importante de ser mencionado é o fato de que, por causa da sarcopenia, muitos idosos deixam de se exercitar em virtude da falta de força, o que gera hábitos cada vez mais sedentários. Nesse sentido, a pessoa tende a permanecer mais tempo sentado ou até acamado, podendo resultar em uma síndrome geriátrica denominada de imobilismo ou na própria síndrome da fragilidade ${ }^{(39)}$. Diante disso, destaca-se o relevante papel da Fisioterapia nesse contexto, com o objetivo de aumentar a amplitude de movimento, promover ganho de força muscular, melhorar o equilíbrio e reduzir os episódios de queda. Todavia deve-se ressaltar que não intervém apenas no tratamento para a sarcopenia, mas também atua eficazmente na prevenção dessa problemática ${ }^{(38)}$.

A liga acadêmica buscou favorecer a troca de conhecimentos e/ou assistência à comunidade, com o surgimento de uma interação entre a academia e a comunidade, sendo de extrema importância por proporcionar a troca de experiência, bem como a união entre ensino e serviço. Além disso, foram desenvolvidas habilidades fundamentais para o ensino e a pesquisa, a oferta de uma assistência integral que fortalece o relacionamento interpessoal e as ações promotoras da saúde, essencial para a qualificação desses profissionais sobre as abordagens empregadas no processo comunicacional, tanto pelas evidências favoráveis que determinam os benefícios na relação interpessoal como pela melhoria na qualidade de vida e saúde desses usuários ${ }^{(40)}$.

Como facilidade da realização da ação destaca-se o fato da LAFIGE ser específica de Gerontologia, o que fez com que fosse mais fácil o entendimento e a compreensão da temática central. Além disso, por ser mediante intermédio acadêmico, houve facilidade também no acesso a acervo literário de qualidade e com respaldo científico.

Como dificuldades destacou-se a falta de investimentos financeiros para a execução da ação pela liga, tendo que ser custeada pelos próprios organizadores. E quanto à limitação, ressalta-se o fato de ter sido realizada apenas em uma praça, não atingindo público mais periférico da cidade. Por isso, recomendam-se outras experiências, com busca de financiamento e recursos por parte da universidade ou governo, além da realização em outros locais, que atingiam público com menor poder aquisitivo e menor acesso a informações.

\section{CONCLUSÃO}

A ação demonstrou-se imprescindível para o desenvolvimento da prática de educação em saúde, a qual é de suma importância para a construção da conscientização da população acerca das alterações que ocorrem com o processo de envelhecimento.

Nesse sentido, a capacitação dos acadêmicos antes de realizar a ação mostrou-se fundamental para efetivar os conhecimentos repassados e, assim, constituir uma relação mais fluída com a população. Desse modo, foi possível auxiliar na edificação e troca de informação para a população desde aspectos conceituais, como a própria avaliação, até o incentivo para a adoção de um estilo de vida saudável. Os ligantes perceberam a importância da prática da fisioterapia em gerontologia e da educação em saúde em busca de uma velhice saudável, a necessidade de conscientização da população sobre o processo de envelhecimento e, a partir do embasamento científico recebido na capacitação, foi possível a troca de saberes de forma bidirecional entre a comunidade e a academia, além do aprimoramento de habilidades sociais e interpessoais dos acadêmicos.

Diante do exposto, o presente estudo propiciou aos acadêmicos da saúde aprendizados e reflexões da importância da extensão universitária no compromisso de retorno científico à comunidade de forma integral, com perspectiva holística e multidimensional, além de perceberem o processo senil como uma fase do ciclo vital, que necessita de atividades completas e que valorizem os conhecimentos de cada participante, visando à promoção da saúde dos idosos. 


\section{CONFLITOS DE INTERESSE}

Os autores declaram a inexistência de conflitos de interesses, tanto na execução das ações do programa de extensão como na escrita deste relato de experiência.

\section{CONTRIBUIÇÕES}

Todos os autores colaboraram em todas as fases da execução das ações e/ou na redação deste relato de experiência.

\section{REFERÊNCIAS}

1. Fried LP. Investing in Health to Create a Third Demographic Dividend. Gerontologist. 2016;56:(S2):167-77.

2. World Health Organization. Decade of Healthy Ageing (2020-2030) [Internet]. 2020 [acesso em 2020 Abr 16]. Disponível em: https://www.who.int/ageing/decade-of-healthy-ageing

3. World Health Organization. World report on ageing and health. Geneva: WHO; 2015.

4. Instituto Brasileiro de Geografia e Estatística. Projeções Populacionais [Internet]. 2020 [acesso em 2020 Abr 16]. Disponível em: https://www.ibge.gov.br/estatisticas/sociais/populacao/9109-projecao-da-populacao. html?=\&t=o-que-e

5. Instituto Brasileiro de Geografia e Estatística. Sistema IBGE de Recuperação Automática - SIDRA. População por grupo de idade, município, $1^{\circ}$ trimestre de 2020 [Internet]. 2020 [acesso em 2020 Abr 16]. Disponível em: https://sidra.ibge.gov.br/tabela/5918\#resultado

6. Instituto Brasileiro de Geografia e Estatística. Síntese de indicadores sociais uma análise das condições de vida da população brasileira. Rio de Janeiro: IBGE; 2016.

7. Mallmann DG, Galindo NM Neto, Sousa JC, Vasconcelos EMR. Educação em saúde como principal alternativa para promover a saúde do idoso. Ciênc Saúde Colet. 2015;20(6):1763-72.

8. World Health Organization. Good health adds life to years Global brief for World Health Day 2012. Geneva: WHO; 2012.

9. Mantovani EP, Lucca SR, Neri AL. Associações entre significados de velhice e bem-estar subjetivo indicado por satisfação em idosos. Rev Bras Geriatr Gerontol. 2016;19(2):203-22.

10. Ministério da Saúde (BR). Política Nacional de Promoção da Saúde (PNPS). Portaria $N^{\circ} 2.446$, de 11 de novembro de 2014 - Redefine a Política Nacional de Promoção da Saúde. Brasília: Ministério da Saúde; 2014.

11. Dátilo GMPA, Cordeio AP. Envelhecimento humano: diferentes olhares. São Paulo: Cultura Acadêmica; 2015.

12. Conselho Federal de Fisioterapia e Terapia Ocupacional. Resolução n 477, de 20 de dezembro de 2016. Reconhece e disciplina a Especialidade Profissional de Terapia Ocupacional em Gerontologia e dá outras providências. Diário Oficial [da] República Federativa do Brasil. Brasília: COFFITO; 2016.

13. Silva LYA, Almeida MCV, Moraes MGB, Carneiro SR. A importância do projeto de extensão na formação acadêmica: relato de experiência de um discente do curso de fisioterapia. Anais do $7^{\circ}$ Congresso de Educação em Saúde da Amazônia (COESA); 2018; Pará. Belém: Universidade Federal do Pará; 2018.

14. Antonio ACFT, Tonhom SFR, Chirelli MQ. Cuidado ao idoso na atenção básica: práticas de educação em saúde do fisioterapeuta. Rev Bras Promoç Saúde. 2016;29(Supl):5-15.

15. Pasqual KK, Rezende KTS, Chirelli MQ. O cuidado prestado pela Estratégia Saúde da Família na percepção das mulheres idosas. Rev Eletrônica Gest Saúde. 2016;(2):685-99.

16. Bendenlaque DFR, Carvalho DNR, Contente RTC. Contribuição da liga acadêmica na formação e produção de conhecimento em saúde do idoso. Braz J Hea Rev. 2019;(2):3762-73.

17. Sociedade Brasileira de Geriatria e Gerontologia. Ligas acadêmicas e residência médica: o que é? [Internet]. [2020] [aceso em 2020 Maio 20]. Disponível em: https://sbgg.org.br/ligas-academicas-e-residencia-medica/oque-e/\# 
18. Castaneda L, Bergmann A, Bahia L. A Classificação internacional de funcionalidade, incapacidade e saúde: uma revisão sistemática de estudos observacionais. Rev Bras Epidemiol. 2014;437-51.

19. Martins R. Envelhecimento, retrogénese do desenvolvimento motor, exercício físico e promoção da saúde. Bol Soc Port Educ Física. 2017;(32):31-40.

20. Amthauer $C$, Falk JW. Discursos dos profissionais de saúde da família na ótica da assistência à saúde do idoso. Res Fundam Care. 2017;9(1):99-105.

21. Malachias MVB, Souza WKSB, Plavnik FL, Rodrigues CIS, Brandão AA, Neves MFT, et al. $7^{\text {a }}$ Diretriz Brasileira de Hipertensão Arterial: capítulo 2-diagnóstico e classificação. Arqu Bras Cardiol. 2016;107(3):7-13.

22. World Health Organization. A guide for population-based approaches to increasing levels of physical activity: implementation of the WHO global strategy on diet, physical activity and health. Geneve: World Health Organization; 2007.

23. Moraes EN. Atenção à saúde do idoso: aspectos conceituais. Brasília: Organização Pan-Americana da Saúde; 2012.

24. World Health Organization. Global recommendations on physical activity for health. Geneve: WHO; 2010.

25. Brasil. Lei $n^{\circ} 8.080$, de 19 de setembro de 1990. Dispõe sobre as condições para a promoção, proteção e recuperação da saúde, a organização e o funcionamento dos serviços correspondentes e dá outras providências [Internet]. 1990 [acesso em 2019 Jun 9]. Disponível em: http://conselho.saude.gov.br/legislacao/ lei8080.htm

26. Formiga LMF, Oliveira EAR, Borges EM, Santos KNC, Araújo AKS, Formiga RCF. Envelhecimento ativo: revisão integrativa. Revista Interdis Ciênc Saúde. 2017;4(2):9-18.

27. Mendonça FTNF, Álvaro SS, Ana LZB, Bruna SSM . Health education with older adults: action research with primary care professionals. Revista Bras Enferm. 2017;70(4):792-9.

28. Carneiro MAP, Silva LFS, Moura TNB, Pereira LCA, Landim MBP. Contribuições da socialização e das políticas públicas para a promoção do envelhecimento saudável: uma revisão de literatura. Rev Bras Promoç Saúde. 2016;29(1):124-31.

29. Viana DA, Martins LC, Gonçalves AM. Educação em saúde como estratégia para promoção do envelhecimento saudável - revisão integrativa da literatura. J Ciên Biomédicas Saúde. 2016;1(3):2.

30. Martins JJ, Barrall DCC, Santosll TM, Hinkell V, Nascimento ERP, Alburquerque GL, et al. Educação em saúde como suporte para a qualidade de vida de grupos da terceira idade. Rev Eletrônica Enferm. 2007;9(2):443-56.

31. Maia FES, Moura ELR, Madeiros EC, Carvalho RRP, Silva SAL, Santos GR. A importância da inclusão do profissional fisioterapeuta na atenção básica de Saúde. Rev Fac Ciênc Méd Sorocaba. 2015;17(3):110-5.

32. Sampaio JF, Dantas CCDBL, Almeida Porto VF, Cavalcante JC, Medeiros ML. A extensão universitária e a promoção da saúde no Brasil: revisão sistemática. Rev Portal Saúde Soc. 2019;3(3):921-30.

33. Barbosa BR, Almeida JM, Barbosa MR, Rossi-Barbosa LAR. Avaliação da capacidade funcional dos idosos e fatores associados à incapacidade. Ciênc Saúde Colet. 2014;19:3317-25.

34. Cavalcanti AL, Moreira RS, Barbosa JMV, Silva VL. Envelhecimento ativo e estilo de vida: uma revisão sistemática da literatura. Estudo Interdisciplin Envelhec. 2016;21(1):71-89.

35. World Health Organization. Envelhecimento ativo: uma política de saúde. Brasília: Organização PanAmericana da Saúde; 2005.

36. Silva FLC, Santana WR, Rodrigues TS. Envelhecimento ativo: o papel da fisioterapia na melhora da qualidade de vida da pessoa idosa: revisão integrativa. Revista Uningá. 2019;56(4):134-44.

37. Malta MB, Papini SJ, Corrente JE. Avaliação da alimentação de idosos de município paulista: aplicação do Índice de Alimentação Saudável. Ciên Saúde Colet. 2013;18:377-84.

38. Souza AF Jr, Silva TPDS, França ICO, Dias GA. Efeitos de um protocolo fisioterapêutico na funcionalidade de idosas institucionalizadas com sarcopenia. Rev Kairós Gerontol. 2018;21(4):191-207. 
39. Oliveira MDJ. Sarcopenia associada ao envelhecimento: fatores que interferem na qualidade de vida do idoso [manuscrito]. 2019.

40. Torres GMC, Figueiredo IDT, Cândido JAB, Pinto AGA, Morais APP, Araújo MFM, et al. Comunicação terapêutica na interação profissional de saúde e hipertenso na estratégia saúde da família. Rev Gaúch Enferm. 2017;38(4)2016-66.

\section{Endereço para correspondência:}

Shaumin Vasconcelos $\mathrm{Wu}$

Universidade Federal do Pará

Rua dos Mundurucus, s/n

Bairro: Guamá

CEP: 66073-000 - Belém - PA - Brasil

E-mail: shauminvwu@gmail.com

Como citar: Wu SW, Fernandes CA, Silva DCF, Nascimento ERS, Campos JS, Oliveira JSS. A importância da liga acadêmica na promoção do envelhecimento saudável. Rev Bras Promoç Saúde. 2020;33:10518. 